\title{
In situ small-angle $x$-ray and nuclear resonant scattering study of the evolution of structural and magnetic properties of an Fe thin film on MgO (001)
}

\author{
Gagan Sharma, ${ }^{1,2}$ Ajay Gupta, ${ }^{1, *}$ Mukul Gupta, ${ }^{2}$ Kai Schlage, ${ }^{3}$ and H.-C. Wille ${ }^{3}$ \\ ${ }^{1}$ Amity Center for Spintronic Materials, Amity University UP, Sector 125, Noida 201 313, India \\ ${ }^{2}$ UGC-DAE Consortium for Scientific Research, University Campus, Khandwa Road, Indore 452 001, India \\ ${ }^{3}$ Deutsches Elektronen-Synchrotron (DESY), Notkestrasse 85, D-22607 Hamburg, Germany \\ (Received 8 May 2015; revised manuscript received 31 July 2015; published 2 December 2015)
}

\begin{abstract}
Growth of magnetron sputtered Fe films on clean single crystalline $\mathrm{MgO}$ (001) substrate has been studied using in situ grazing incidence small angle x-ray scattering (GISAXS) and grazing incidence nuclear resonant scattering (GINRS) measurements. While GISAXS provides information about morphological changes, GINRS provides information about structural and magnetic properties, thus making it possible to correlate the evolution of magnetic properties with that of morphology and structure of the film. The film exhibits a Volmer-Weber type growth, with percolation transition occurring around $2 \mathrm{~nm}$ film thickness. Presence of a finite quadrupole splitting, as seen in GINRS measurements, suggests a significant distortion from cubic symmetry up to a film thickness of $3.5 \mathrm{~nm}$, which can be attributed to hybridization between $\mathrm{Fe} 3 d$ and $\mathrm{O} 2 p$ orbitals at the interface as well as in-plane tensile strain induced as a result of coalescence of islands. Initially Fe islands exhibit superparamagnetic relaxation, while finite magnetic moment appears upon formation of macroscopic percolation islands. The film exhibits a weak perpendicular magnetic anisotropy (PMA), which vanishes concurrently with disappearance of structural distortion, suggesting that the observed PMA at least partly originates from inherent strain in the film. No presence of any known oxide of Fe was detected at the interface. More precise information about topological and magnetic structure of the interfaces between $\mathrm{Fe}$ and $\mathrm{MgO}$ layers is obtained using combined x-ray reflectivity and nuclear resonance reflectivity measurements on a ${ }^{57} \mathrm{Fe} / \mathrm{MgO}$ multilayer. Measurements show that about two monolayers of $\mathrm{Fe}$ at the interface have a reduced hyperfine field, providing evidence for hybridization with $\mathrm{O}$ atoms, as predicted by theory.
\end{abstract}

DOI: 10.1103/PhysRevB.92.224403

PACS number(s): 75.70.-i, 68.35.Ct, 68.55.J-, 76.80.+y

\section{INTRODUCTION}

Magnetic tunnel junctions (MTJs) consisting of two ferromagnetic layers separated by a thin insulating tunnel barrier have generated great scientific and technological interest due to their potential applications in magnetic recording heads, nonvolatile random access memories, logic devices, etc. Realization of symmetry filtering effect in the case of crystalline $\mathrm{MgO}$ (001) barrier layers resulted in a remarkable boost in the maximum tunnel magneto resistance (TMR) value. The highest TMR of $\sim 600 \%$ has been observed in the $\mathrm{CoFeB} / \mathrm{MgO} / \mathrm{CoFeB}$ system [1]. However, these values still fall significantly short of the theoretically predicted value of $\sim 1000 \%$ in epitaxial $\mathrm{Fe} / \mathrm{MgO} / \mathrm{Fe} \mathrm{MTJ}$ [2]. Interfacial imperfections in terms of possible oxidation of transition metal in the interfacial region or oxygen vacancies, etc., are suggested to be possible reasons for deterioration in TMR value [3-5]. This has led to extensive study in the literature on characterization of interfaces in $\mathrm{MgO}$ based MTJs [6-11]. However, understanding of various interfacial imperfections and their correlation with magnetic and magneto-transport properties of magnetic electrodes is still far from satisfactory.

From the point of view of applications in nonvolatile magnetic random access memory devices, it is widely recognized that current induced spin transfer torque (STT) will be used for switching the magnetization of the free magnetic layer in a MTJ. However, the level of current density needed to reorient the magnetization is presently too high to be usable

\footnotetext{
*agupta2@amity.edu
}

in commercial applications. Recently it has been realized that the threshold current density can be significantly reduced by introducing perpendicularly magnetized electrodes in MTJs $[12,13]$. The MTJ with magnetic electrode having perpendicular magnetic anisotropy (PMA) are expected to have superior properties both in terms of lower switching current density as well as higher thermal stability [12-15]. However, conventional PMA materials such as $\mathrm{Co} / \mathrm{Pt}$ multilayer or $\mathrm{L}_{0}$ ordered (Co, Fe) Pt alloys are not suitable for such applications due to low spin polarization. This has led to a spurt in exploration of systems with PMA, which could be used as a magnetic electrode in perpendicular anisotropy MTJs $[8,9,12,16]$. Ultrathin films of Fe, bcc-CoFe alloy, or amorphous $\mathrm{CoFeB}$ on $\mathrm{MgO}$ have been found to possess perpendicular anisotropy $[8,9,12]$. Studies of PMA in these systems as a function of film thickness suggest that PMA mainly originates at the interface [17,18]. This interfacial PMA is generally interpreted in terms of a strong hybridization effect between $\mathrm{Fe}(\mathrm{Co}) 3 d$ orbital pointing toward the interface (viz. $d_{\mathrm{z}}^{2}, d_{\mathrm{zx}}, d_{\mathrm{yz}}$ ) with $\mathrm{O} 2 p_{\mathrm{z}}$ orbitals [11,12,19-21]. This hybridization combined with spin-orbit coupling is suggested to result in observed PMA. However, a number of first principle calculations suggest that oxygen content in the interfacial region has only a small effect on PMA [20,22]. Thus, the origin of PMA seems to be more complex in nature. He and Chen also studied the effect of strain on PMA in $\mathrm{FeCo} / \mathrm{MgO}$ (001) using first principle calculations [23]. It was found that variation of in-plane lattice constants resulted in switching of the direction of magnetic anisotropy.

It may be mentioned that although from an application point of view, amorphous $\mathrm{CoFeB}$ is the material of choice 
for magnetic electrodes, presence of boron in the interfacial region adds to the complexity of the system, making it difficult to model the system theoretically. Therefore, from the point of view of basic understanding, most of the studies, both theoretical as well as experimental, have been done on simpler systems such as bcc-Fe or FeCo [8,11,19,23,24]. Systematic study of PMA as a function of Fe film thickness in $\mathrm{MgO} / \mathrm{Fe} / \mathrm{MgO}$ trilayer system has been done using nuclear resonant scattering (NRS) and conversion electron Mössbauer spectroscopy (CEMS) [10,25]. In both of the studies, existence of PMA at low temperatures has been confirmed. The PMA is found to decrease with increasing film thickness. Systematic measurements suggest that PMA has an interfacial origin, as predicted by theory $[19,26]$. Balogh et al. attributed the perpendicular anisotropy to mechanical stresses in the film [25], arising from the epitaxial relation and different temperature dilation of various layers; however, no direct evidence in support of this claim was provided.

Furthermore, PMA is observed only in thin films in the range of a few nanometer thicknesses. In view of the ultrathin nature of the film, its magnetic properties can also get affected significantly by its growth behavior. Therefore, in the present paper, in situ grazing incidence small angle X-ray scattering (GISAXS) and grazing incidence NRS (GINRS) techniques have been used to study the growth behavior of thin Fe film on $\mathrm{MgO}$ and the evolution of its structural and magnetic properties with film thickness. Such combination of in situ GISAXS and GINRS techniques has earlier been used to study the evolution of structural and magnetic properties of iron nanostructures on ordered polymer templates [27]. While GISAXS provides morphological information, GINRS provides information about the evolution of both structural as well as magnetic properties through hyperfine interactions.

\section{EXPERIMENTAL}

Single crystalline polished $\mathrm{MgO}(001)$ substrate was used to study the growth behavior of thin Fe film. In order to improve the surface quality, $\mathrm{MgO}$ substrate was given the following treatment: The as-received substrate was annealed at $700^{\circ} \mathrm{C}$ for $1 \mathrm{~h}$ in a vacuum of $2 \times 10^{-6}$ mbar followed by ion etching using $\mathrm{Ar}$ ions at $500 \mathrm{eV}$ energy for $90 \mathrm{~s}$ at a flux of $1.5 \times$ $10^{16}$ ions $\mathrm{cm}^{-2} \mathrm{~s}^{-1}$, followed by another vacuum annealing at $700^{\circ} \mathrm{C}$ for $30 \mathrm{~min}$. This treatment is known to improve surface quality and reduce carbon contamination, and such surfaces have been used in the literature to deposit good quality films [28-30]. Fe film on such substrate was deposited using the technique of magnetron sputtering, which has been used quite often in the literature to deposit TMR multilayers [31,32]. Growth behavior of the film was studied in situ using GISAXS and GINRS measurements. The experiments were performed at P01 beamline of PETRA III [33], which has the possibility of doing simultaneous GISAXS and GINRS measurements. During measurements, the synchrotron was operating in the 40 bunch mode with bunch separation of 192 ns. For in situ measurements, $\mathrm{MgO}$ (001) substrate was loaded in an ultrahigh vacuum chamber, which was positioned in the path of the beam and was mounted on a Huber goniometer so as to vary the angle of incidence. Substrate was mounted in such a way that its (100) axis was along the x-ray propagation direction. The chamber is equipped with the facility of dc magnetron sputtering for thin film deposition. The GISAXS pattern was recorded on an MAR 345 image plate, which was kept at a distance of around $4.034 \mathrm{~m}$ from the chamber. In order to avoid absorption and stray scattering by air, the path of beam the sample and image plate was evacuated. GINRS measurements were done using an avalanche photodiode (APD) detector, which was placed downstream from the image plate.

Prior to deposition, the substrate was preheated in ultrahigh vacuum (UHV) at a moderate temperature of $150^{\circ} \mathrm{C}$ for $30 \mathrm{~min}$ in order to remove possible adsorbed water at the surface. The base pressure in the chamber was $2 \times 10^{-7}$ mbar. Deposition of the ${ }^{57} \mathrm{Fe}$ (95\% enriched) film was carried out using the dc magnetron sputtering at $6 \mathrm{~W}$ power, with the substrate kept at room temperature. The sputtering power was chosen on the basis of precalibration, according to which a deposition rate of $0.01 \mathrm{~nm} / \mathrm{s}$ was expected at this power. The deposition rate was cross-checked by taking $\mathrm{X}$-ray reflectivity (XRR) in situ at the end of the deposition cycle of $450 \mathrm{~s}$. No magnetic field was applied to the sample during deposition or during measurements.

In situ measurements of both GINRS and GISAXS were performed with increasing film thickness at nuclear resonance energy of ${ }^{57} \mathrm{Fe}$, i.e., $14.4 \mathrm{keV}$. The $\mathrm{x}$-ray beam was allowed to fall on the specimen at a grazing angle of $0.25^{\circ}$ with its polarization vector (electric field vector) in the film plane. After depositing a predesignated film thickness, deposition was stopped, and the GISAXS pattern was recorded. For the GINRS measurement, the image plate was removed from the path of the scattered beam. One set of GISAXS and GINRS measurements took about $10 \mathrm{~min}$, after which the deposition was resumed. Measurements were done as a function of deposition time from $100 \mathrm{~s}$ to $450 \mathrm{~s}$, with a step of $50 \mathrm{~s}$.

In order to get more detailed information about the $\mathrm{Fe} / \mathrm{MgO}$ interface, nuclear resonance reflectivity (NRR) measurements were done on a ${ }^{57} \mathrm{Fe} / \mathrm{MgO}$ multilayer having nominal structure $\mathrm{Si}$ (substrate) $/\left[{ }^{57} \mathrm{Fe}(2.5 \mathrm{~nm}) / \mathrm{MgO}(2.5 \mathrm{~nm})\right]_{\times 10}$. Fe layers were prepared with $95 \%$ enriched ${ }^{57} \mathrm{Fe}$ target in order to enhance the NRR signal. The thicknesses of $\mathrm{Fe}$ and $\mathrm{MgO}$ layers were intentionally kept equal. Deposition was carried out at room temperature in a UHV chamber equipped with the facility of ion beam sputtering using $3 \mathrm{~cm}$ Kaufman type ion source. The base vacuum of the chamber was $2 \times 10^{-7} \mathrm{mbar}$. Sputtering was done with $1 \mathrm{keV}$ Ar ions with a beam current of $25 \mathrm{~mA}$. The NRR in time integral mode was carried out on ${ }^{57} \mathrm{Fe} / \mathrm{MgO}$ multilayer at the P01 beamline itself in the presence of a magnetic field of 200 Oe, applied in the sample plane perpendicular to propagation vector, so as to align the magnetic moment of $\mathrm{Fe}$ along polarization vector. The XRR of the multilayer was measured using Bruker D8 diffractometer fitted with a Göbel mirror on the incident beam side in order to obtain a parallel monochromatic beam of the $\mathrm{Cu} K_{\alpha}$ radiation.

\section{RESULTS AND DISCUSSION}

Figure 1 gives the specular XRR of the film taken in situ after the final deposition of $450 \mathrm{~s}$. The $\theta-2 \theta$ scan was taken using the APD detector. Fitting of the data using Parratt's 


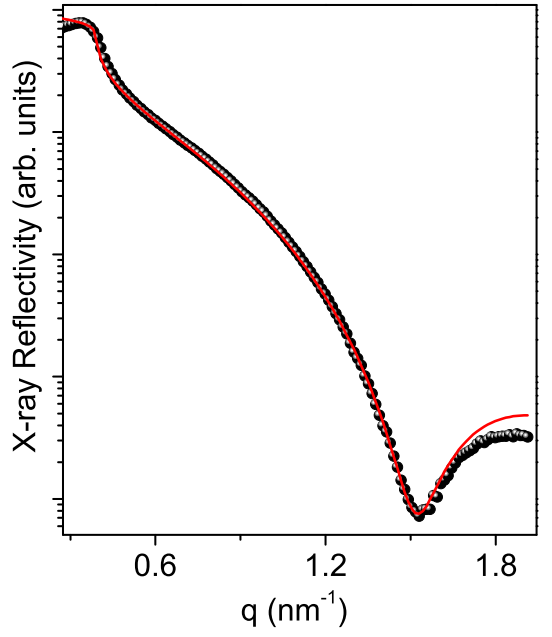

FIG. 1. (Color online) X-ray reflectivity of an Fe film taken in situ at the end of the deposition for $450 \mathrm{~s}$. Continuous curve represents the best fit to experimental data.

formalism yielded a film thickness of $4.5 \mathrm{~nm}$ and surface and interface roughness of $0.4 \mathrm{~nm}$ and $0.6 \mathrm{~nm}$, respectively. Film thickness matches very well with the nominal value expected on the basis of predetermined deposition rate.

\section{A. GISAXS study}

GISAXS measurements were done in order to study evolution of film morphology as a function of thickness. It may be noted that $\mathrm{MgO}$ has a significantly lower surface free energy $\left(1.1 \mathrm{~J} / \mathrm{m}^{2}\right)$ as compared to $\mathrm{Fe}\left(2.9 \mathrm{~J} / \mathrm{m}^{2}\right)$ [34]. Therefore, one expects a Volmer-Weber type of growth. Initially the film would consist of $\mathrm{Fe}$ islands on $\mathrm{MgO}$. With increasing film thickness, percolation transition is expected to occur, with appearance of macroscopic island.

Figure 2 shows a typical 2D GISAXS pattern of a $4.5 \mathrm{~nm}$ thick Fe film on $\mathrm{MgO}$ (001). A horizontal strip around $q_{\mathrm{z}}=0.55 \mathrm{~nm}^{-1}$ was extracted in order to get the scattered intensity as a function of in-plane scattering vector $q_{\mathrm{y}}$ for GISAXS analysis. A vertical strip around $q_{\mathrm{y}}=0 \mathrm{~nm}^{-1}$ was used to extract scattered intensity as a function of scattering vector $q_{\mathrm{z}}$. Figure 3 gives the GISAXS data (scattered intensity vs $q_{\mathrm{y}}$ ) for different film thicknesses. The data was analyzed using software FitGISAXS [35]. The model used for fitting consisted of islands of a spherical shape separated by an average distance $\Lambda$, defined as the distance between two neighboring island centers. Islands were assumed to have a lognormal distribution of diameter, while the interisland separation was assumed to have a Gaussian distribution. The average island size (which is also the height of island) was determined from the position of minimum in the vertical cut. The average interisland distance $(\Lambda)$, width of its distribution, and width of particle size distribution were taken as fitting parameters. In order to match the simulated pattern with the experimental data, it was necessary to multiply it with a scale factor. This scale factor would, in general, depend upon factors like photon flux, time of measurement, and fractional area covered by islands, etc. Scale factor was also taken as a fitting parameter. This model is in accordance with some
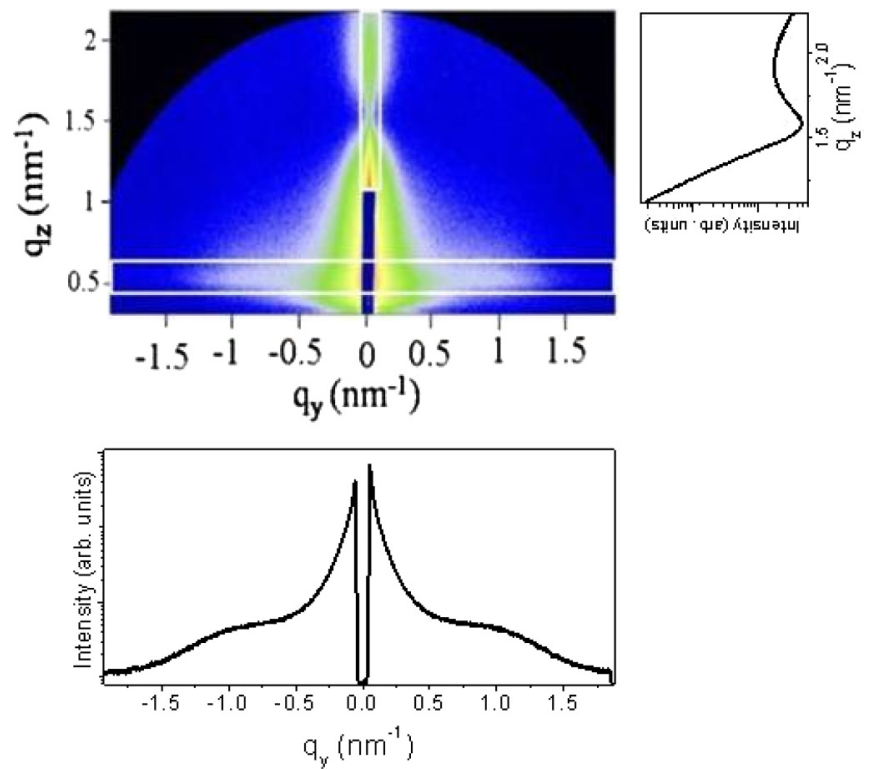

FIG. 2. (Color online) 2D GISAXS pattern of $4.5 \mathrm{~nm}$ thick Fe film on $\mathrm{MgO}$ (001). A horizontal strip around $q_{\mathrm{z}}=0.55 \mathrm{~nm}^{-1}$ was extracted for the GISAXS analysis. The vertical strip around $q_{\mathrm{y}}=$ $0 \mathrm{~nm}^{-1}$ was extracted for island height determination.

earlier studies on thin $\mathrm{Fe}$ film on $\mathrm{MgO}$ using GISAXS or STM measurements [36,37], where shape of Fe islands in as-deposited film was found to be spherical.

Up to a film thickness of $2.5 \mathrm{~nm}$, this model gave a reasonably good fit. Beyond this thickness, it became necessary to take islands of prolate spheroidal shape, with vertical axis being a few percent larger than the in-plane axes. Results of fitting are shown in Fig. 4. It may be noted that as long as the islands are well separated from each other, their shape remains spherical within experimental error. Once the islands start touching/overlapping, the shape distorts and become spheroidal. It is interesting to see that the scale factor exhibits a decrease with increase in film thickness. Since the GISAXS data shown in Fig. 4 was normalized with time of measurement and the photon flux on the sample was constant, the observed variation in scale factor cannot be attributed to these aspects. On the other hand, the fractional area covered by the islands can change as a result of formation of percolating clusters. Considering the lower $q$ value accessible in the present experiment $\left(\sim 0.08 \mathrm{~nm}^{-1}\right)$, islands of a size bigger than a few tens of $\mathrm{nm}$ will contribute to scattering intensity below the accessible $q$ range. Thus, as the islands coalesce and acquire size more than a few tens of $\mathrm{nm}$, they will not contribute to scattering intensity in the $q$ range used in the present experiment. This will result in the decrease in the scale factor. A rapid decrease in the scale factor up to a thickness of $2 \mathrm{~nm}$ suggests that percolation transition is taking place in this thickness range, where islands coalesce to form macroscopic ones.

\section{B. GINRS study}

Figure 5 gives the GINRS spectra of Fe film as a function of thickness. There is a significant variation in the beat pattern 


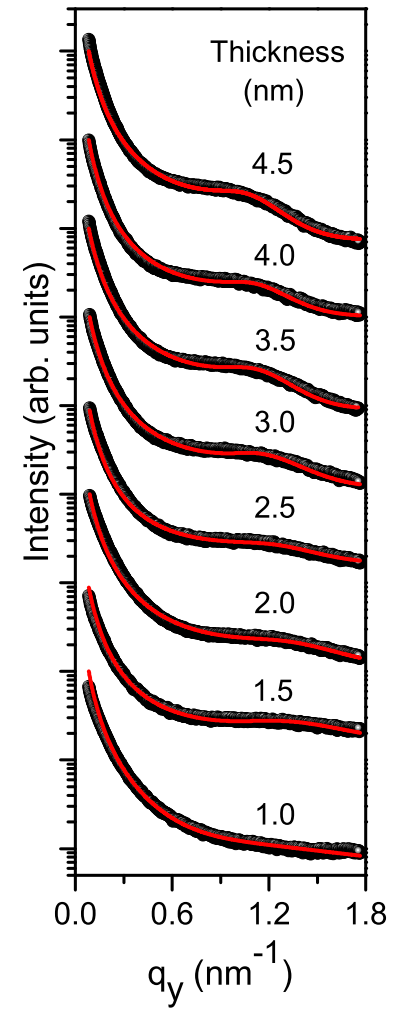

FIG. 3. (Color online) GISAXS data as a function of film thickness. Continuous curves represent the best fit to experimental data. Different curves are displaced vertically with respect to each other for easy viewing.

with increasing film thickness. Oscillations in the time spectra can originate due to the splitting of the nuclear hyperfine level of ${ }^{57} \mathrm{Fe}$ as a result of the interaction of the quadrupole moment of the nucleus with electric field gradient due to the surrounding electronic charges, as well as due to the magnetic dipole interaction of nuclear magnetic dipole moment with internal hyperfine magnetic field $\left(B_{\mathrm{hf}}\right)$.

For a lattice with cubic symmetry, the electric field gradient due to surrounding ligands will be zero; therefore, the quadrupole splitting (QS) will also be zero. Any deviation from cubic symmetry gives rise to a finite electric field gradient and, hence, a finite QS. Thus, information about local structural symmetry around the Fe atom can be obtained from QS. The detailed shape of the beat pattern not only depends on the magnitude of the internal hyperfine field but also upon its direction with respect to the propagation vector of $\mathrm{x}$ rays [38]. Thus, the fitting of GINRS spectra can yield structural information through QS as well as the information about the magnitude and direction of atomic magnetic moments. Fitting of GINRS data was carried out using the software REFTIM [39].

Broad oscillations in the time spectra up to a thickness of $1.5 \mathrm{~nm}$ could be fitted with a finite QS only. For film thickness of $2 \mathrm{~nm}$ or more, additional short period oscillations start appearing due to magnetic hyperfine interactions. Accordingly, various fitting parameters used in the analysis are QS, hyperfine field $\left(B_{\mathrm{hf}}\right)$, width of hyperfine field distribution $\left(\Delta B_{\mathrm{hf}}\right)$, and the direction of the magnetic hyperfine field,



FIG. 4. Variation of scale factor normalized with measurement time, average diameter of islands $(D)$, average height of islands $(H)$, and the average interisland distance $(\Lambda)$, as obtained from fitting of GISAXS data as a function of film thickness.

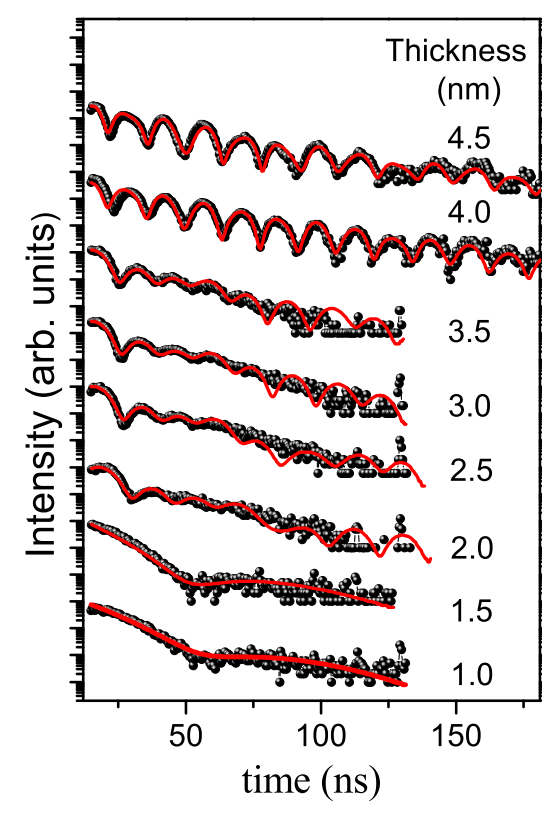

FIG. 5. (Color online) GINRS time spectra of Fe film as a function of film thickness. Continuous curves represent the best fit to experimental data. Different curves are displaced vertically with respect to each other for easy viewing. 
which can be defined in terms of two angles, namely, angle $\beta$ with respect to the surface normal and the azimuthal angle $\gamma$ with respect to the direction of polarization of $\mathrm{x}$ rays. $\Delta B_{\mathrm{hf}}$ represents the distribution of hyperfine fields around the mean value $B_{\mathrm{hf}}$. Since one expects a distribution in island size, $B_{\mathrm{hf}}$ could also exhibit a distribution because of variation in thermal fluctuations in the magnetic moment of an island. This is taken care of by $\Delta B_{\mathrm{hf}}$. The hyperfine field was assumed to have a Gaussian distribution, with $\Delta B_{\mathrm{hf}}$ being its standard deviation. Since Fe film on $\mathrm{MgO}(001)$ has an in-plane crystallographic relation Fe [100]||MgO [110], the azimuthal angle $\gamma$ was therefore constrained to be around $45^{\circ}$ [the sample was mounted with its $\mathrm{MgO}$ (100) axis roughly along the propagation vector]. A small variation in the value $\gamma$ was considered in order to optimize the fitting. This variation in $\gamma$ could be due to some misalignment of the sample with respect to the beam. The direction of the electric field gradient responsible for QS was taken to be parallel to that of $B_{\mathrm{hf}}$. The sign of QS comes out to be positive. In the case of ${ }^{57} \mathrm{Fe}$, since quadrupole moment is positive, a positive QS means a positive field gradient. An almost in-plane electric field gradient is consistent with in-plane tensile strain, as such a strain would try to generate electric field gradient with its principal axis in the film plane [40]. The results of fitting are summarized in Fig. 6.

From Fig. 6, one may note that up to a thickness of $1.5 \mathrm{~nm}$, the hyperfine field is zero, suggesting that the film is nonmagnetic. In this thickness range, average island size, as obtained from GISAXS, varies from $2 \mathrm{~nm}$ to $3 \mathrm{~nm}$, which is well within the superparamagnetic relaxation limit of Fe. Thus, absence of magnetism can be understood in terms of

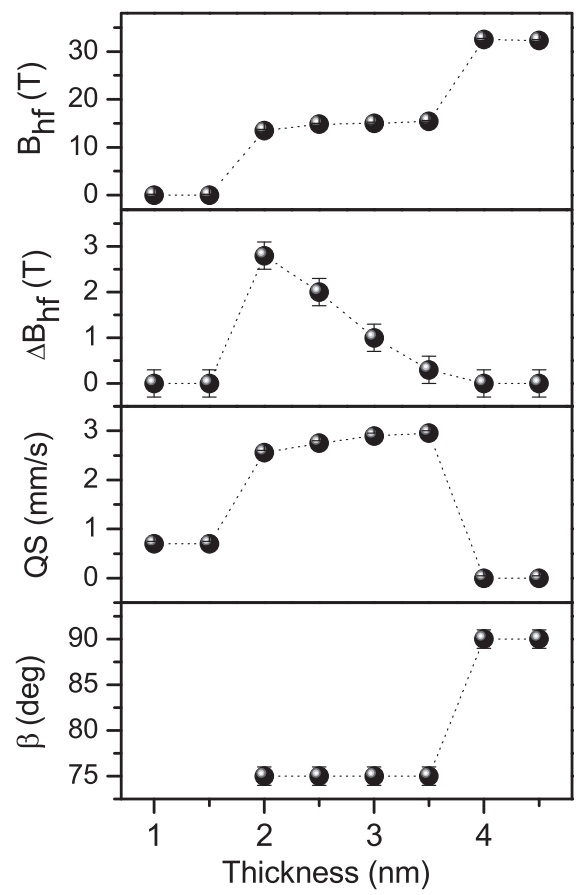

FIG. 6. Variation of hyperfine field $\left(B_{\mathrm{hf}}\right)$, width of hyperfine field distribution $\left(\Delta B_{\mathrm{hf}}\right)$, quadrupole splitting (QS), and average angle of atomic spins with respect to surface normal $(\beta)$ as a function of film thickness. superparamagnetic relaxation of islands. Superparamagnetic relaxation occurs when the magnetic anisotropy energy of a single domain particle is comparable to thermal energy. Thermal fluctuations make the magnetization of the particle fluctuate along different easy magnetization directions. In the case that the fluctuation rate of the magnetization direction due to superparamgnetic relaxation is higher than the Larmor precession rate of Fe nuclei, the hyperfine field averages out to zero [41]. The presence of superparamagnetic relaxation in thin films of $\mathrm{Fe}$ on $\mathrm{MgO}$ has also been seen in some earlier NRS and CEMS studies [10,25]. At a thickness of $2.0 \mathrm{~nm}$, the film exhibits a finite hyperfine field. With further increase in thickness, it exhibits a slow increase. It may be noted that once macroscopic islands form as a result of percolation transition, relaxation of their magnetic moments will slow down, resulting in a finite hyperfine field. Thus, GINRS measurements suggest that percolation transition takes place around a thickness of $2 \mathrm{~nm}$. This is in conformity with the results of GISAXS measurements. At a thickness of $4.0 \mathrm{~nm}$, the film exhibits a hyperfine field value of $33 \mathrm{~T}$, which corresponds to bulk Fe.

It may be mentioned that for cubic symmetry the electric filed gradient and, hence, QS is expected to be zero. Thus, a finite QS value suggests a distortion from cubic structure. Film thickness dependence of QS can be understood in terms of strain generated in the film during growth. There can be several reasons for this strain generation. It has been suggested that hybridization of atomic orbitals of metal and oxygen atoms at the interface results in elastic distortion of crystal lattice of both magnetic layer as well as $\mathrm{MgO}$, which can induce an in-plane tensile strain $[20,42]$. In-plane tensile strain can also be generated due to coalescence of islands [43]. An initially small QS seen up to a thickness of $1.5 \mathrm{~nm}$ may be attributed primarily to strain generated as a result of hybridization at the interface. Formation of macroscopic percolating islands at a film thickness of $2 \mathrm{~nm}$ evidences abundant coalescence of islands. This would result in further generation of in-plane tensile strain, causing QS to increase rapidly.

The angle $\beta$ between the film surface normal and direction of hyperfine field (which is the same as that of atomic magnetic moment) is about $75^{\circ}$ for film thickness up to $3.5 \mathrm{~nm}$, suggesting the presence of small PMA. However, for a film thickness of $4.0 \mathrm{~nm}, \beta$ goes to $90^{\circ}$, signaling that the magnetic moment becomes parallel to film surface. Thus, at this thickness the PMA disappears. It is interesting to note that the film exhibits a PMA as long as its structure deviates from cubic symmetry, as evidenced by a finite value of QS. As the QS goes to zero and the film attains cubic symmetry, the PMA also disappears. This observation provides clear evidence that structural distortion in thin film is at least partly responsible for the observed PMA. Thus, present GINRS measurements suggest that the observed PMA in Fe on $\mathrm{MgO}$ (001) has contributions from both interfacial hybridization as well as strain generated in the bulk of film due to coalescence and possible epitaxial strain. It has been seen that interfacial contribution is significant only up to the thicknesses of the order of $1 \mathrm{~nm}$ [18]. Thus, in the present case PMA seen up to a thickness of $3.5 \mathrm{~nm}$ is expected to have a dominant contribution from bulk tensile stress.

In order to achieve a good fitting of the time spectra of films of thicknesses $\geqslant 4.0 \mathrm{~nm}$, it was necessary to introduce a 


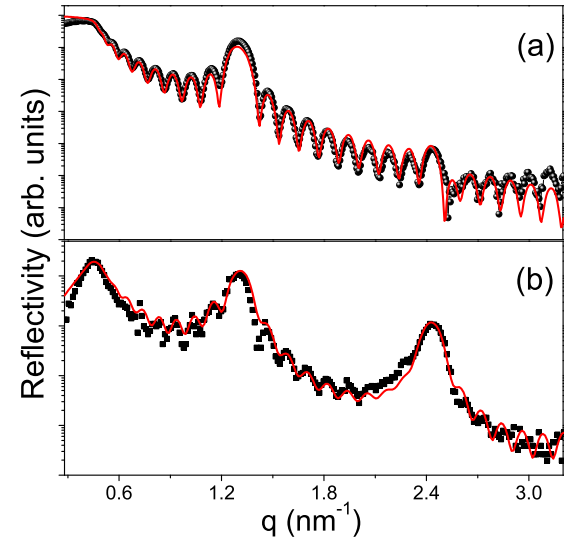

FIG. 7. (Color online) (a) X-ray reflectivity of the $\left[{ }^{57} \mathrm{Fe} / \mathrm{MgO}\right]_{10}$ multilayer taken at an energy of $8 \mathrm{keV}$. (b) Nuclear resonance reflectivity of the same taken at nuclear resonance energy of ${ }^{57} \mathrm{Fe}$, i.e., $14.4 \mathrm{keV}$. Continuous curves represent the best fit to the experimental data. Different curves are displaced vertically with respect to each other for easy viewing.

thin interfacial layer of reduced hyperfine field. Best fit was obtained when an interfacial layer having hyperfine field of $30.8 \mathrm{~T} \pm 0.1 \mathrm{~T}$ and thickness of about $0.4 \mathrm{~nm} \pm 0.2 \mathrm{~nm}$ was introduced. This result is consistent with our earlier GINRS study of Fe film on $\mathrm{MgO}$ (001) [29]. In order to obtain more precise information about the interfacial layer of the reduced hyperfine field, NRR measurements were done on a multilayer of ${ }^{57} \mathrm{Fe} / \mathrm{MgO}$.

\section{NRR study}

Multilayer having nominal structure $\left[{ }^{57} \mathrm{Fe}(2.5 \mathrm{~nm}) / \mathrm{MgO}\right.$ $(2.5 \mathrm{~nm})]_{\times 10}$ was used for the NRR study. The thicknesses of $\mathrm{Fe}$ and $\mathrm{MgO}$ layers were intentionally kept equal. Figure 7(a) gives the XRR of the film taken at energy of $8 \mathrm{keV}$. Electron density contrast between $\mathrm{Fe}$ and $\mathrm{MgO}$ layers gives rise to Bragg peaks in the reflectivity corresponding to the periodicity of the multilayer. One can see that the second order Bragg peak is almost absent, which is as per expectation due to almost equal thicknesses of $\mathrm{Fe}$ and $\mathrm{MgO}$ layers. A detailed fitting of the XRR gives the thicknesses of the two layers as $d_{\mathrm{Fe}}=2.5 \pm$ $0.1 \mathrm{~nm}$ and $d_{\mathrm{MgO}}=2.7 \pm 0.1 \mathrm{~nm}$ and their interface roughness as $\sigma_{\mathrm{Fe}}=0.6 \pm 0.05 \mathrm{~nm}$ and $\sigma_{\mathrm{MgO}}=0.55 \pm 0.05 \mathrm{~nm}$.

NRR on the same multilayer was measured at a nuclear resonance energy of ${ }^{57} \mathrm{Fe}$ [44] and is shown in Fig. 7(b). It is interesting to note that in contrast to the XRR, NRR exhibits a strong second order Bragg peak despite the thicknesses of $\mathrm{Fe}$ and $\mathrm{MgO}$ layers being almost equal. At grazing incidence geometry, the index of refraction of the layer material can be written as [44]

$$
\begin{aligned}
n_{a b} & =\sqrt{1+\frac{\lambda_{0}^{2}}{\pi} \sum_{i} \sigma_{i}\left(f_{i}\right)_{a b}} \\
& \approx 1+\frac{\lambda_{0}^{2}}{2 \pi} \sum_{i} \sigma_{i}\left[\left(f_{i}^{e}\right)_{a b}+\left(f_{i}^{n}\right)_{a b}\right],
\end{aligned}
$$

where $\left(f_{i}^{e}\right)_{a b}$ and $\left(f_{i}^{n}\right)_{a b}$ are, respectively, the electronic and nuclear scattering amplitudes for scattering $\overrightarrow{\varepsilon_{b}}$ polarized radiation into $\overrightarrow{\varepsilon_{a}}$ polarized radiation and $\sigma_{i}$ is the atomic density of the species $i$. For forward scattering away from the absorption edge for an isotropic dipole oscillator and no polarization mixing, the electronic scattering amplitude is given by

$$
\left(f^{e}\right)_{a b}=\delta_{a b} f^{e}=-Z r_{e}+\frac{i \sigma_{e}}{4 \pi \lambda_{o}},
$$

where $r_{e}=\mathrm{e}^{2} / m c^{2}$ is the classical radius of the electron and $\sigma_{e}$ is the photoelectric cross-section. Thus, the electronic contribution to the refractive index has only a weak dependence on the $\mathrm{x}$-ray energy. For the case of $2^{\mathrm{L}}$-pole transition, the resonant scattering amplitude is given by [45]

$$
\left(f_{n}\right)_{a b}=\frac{4 \pi f_{\mathrm{LM}}}{k_{0}} \sum_{M=-L}^{L}\left[\varepsilon_{a} \cdot Y_{\mathrm{LM}}\left(k_{0}\right)\left[Y_{\mathrm{LM}}^{*}\left(k_{0}\right) \cdot \varepsilon_{b}\right] F_{\mathrm{LM}}(\omega),\right.
$$

where $f_{\mathrm{LM}}$ is the Lamb-Mössbauer factor and $Y_{\mathrm{LM}}\left(k_{0}\right)$ are the vector spherical harmonics. A strong energy dependence of resonance scattering amplitude comes from $F_{\mathrm{LM}}(\omega)$, which is given by

$$
F_{\mathrm{LM}}(\omega)=\sum_{\alpha, \eta} \frac{p_{\alpha} p_{\alpha}(\eta) \Gamma_{x}(\alpha M \eta ; L)}{[E(\eta)-E(\alpha)-\hbar \omega]-i \Gamma(\eta) / 2} .
$$

The sum runs over all initial states $\alpha$ and the excited states $\eta \cdot p_{\alpha}$ is the probability that the initial state $\alpha$ is occupied, and $p_{\alpha}(\eta)$ is the probability that the state $\eta$ is initially unoccupied. $\hbar \omega$ is the photon energy, while $E(\alpha)$ and $E(\eta)$ are the energies of the ground state and excited levels, respectively. $\Gamma_{x}(\alpha M \eta ; L)$ is the partial resonance width of the transition between $\alpha$ and $\eta$ with a change of $M$ in the magnetic quantum number; $\Gamma(\eta)$ denotes the full resonance width.

One can see that the nuclear resonance term depends upon the nuclear transition energy and therefore will exhibit a variation with possible variation in hyperfine splitting. Therefore, a possible interfacial layer with reduced hyperfine field will have a different NRS cross section as compared to the bulk of Fe layer. Therefore, the NRR pattern has to be fitted by taking a four layer model, with the two additional layers being the reduced hyperfine field layers at the two interfaces. The NRR was also fitted using the software REFTIM [39]. The overall thicknesses of $\mathrm{Fe}$ and $\mathrm{MgO}$ layers were taken to be the same, as obtained from the fitting of the XRR pattern. The hyperfine field of the interfacial layer was taken from the fitting of GINRS spectra. Therefore, the only independent fitting parameter in this case was the thickness of the interfacial layer. The best fit was obtained for a thickness of interfacial layer equal to $0.24 \pm 0.05 \mathrm{~nm}$. This thickness is roughly equal to 2 monolayers of Fe.

It may be interesting to compare the present results with some earlier CEMS studies on the Fe/MgO system. Zając et al. studied $5 \mathrm{ML}$ thick ${ }^{57} \mathrm{Fe}$ on $\mathrm{MgO}(001)$ in situ in a UHV chamber using CEMS [46]. While at room temperature, the Fe film was nonmagnetic due to superparamgnetic relaxation; at $80 \mathrm{~K}$, it exhibited magnetically split spectrum. The $80 \mathrm{~K}$ spectrum could be fitted reasonably well with two overlapping 
broad sextets. From the relative area of two hyperfine field components, it was concluded that about 2 MLs have a reduced hyperfine field of $29.7 \mathrm{~T}$. At room temperature, the interfacial component was also found to exhibit a large QS of $0.47 \mathrm{~mm} / \mathrm{s}$. Our results on the interfacial layer shows a reduced hyperfine field of $30.8 \mathrm{~T}$, which is in good agreement with their results, as well as with an earlier GINRS study of the ${ }^{57} \mathrm{Fe}-\mathrm{on}-\mathrm{MgO}$ (001) interface [29].

In contrast to these studies, both CEMS and NRS studies on the $\mathrm{MgO} / \mathrm{Fe} / \mathrm{MgO}$ trilayer system suggest a presence of two interfacial components: one with reduced hyperfine field and the other with hyperfine field greater than that of bulk Fe. Furthermore, in an earlier Mössbauer study on the ${ }^{57} \mathrm{Fe}$ layer covered with $\mathrm{MgO}$, a single interfacial component with an enhanced hyperfine field was observed [47]. It is interesting to note that a higher hyperfine field component is observed in two of the above studies where an $\mathrm{MgO}$ overlayer is grown on Fe film. These results can be reconciled by conjecturing that $\mathrm{Fe}-\mathrm{on}-\mathrm{MgO}$ and $\mathrm{MgO}$-on-Fe interfaces behave differently and that the interfacial layer at $\mathrm{MgO}$-on-Fe interface exhibits enhancement of a hyperfine field, while at the Fe-on-MgO interface, the hyperfine field gets reduced. In the present paper as well as systems studied in Refs. [29] and [46], only the $\mathrm{Fe}-\mathrm{on}-\mathrm{MgO}$ interface exists, while in Ref. [47] only the $\mathrm{MgO}-$ on-Fe interface exists. Indeed there are experimental evidences to show that the two $\mathrm{Fe} / \mathrm{MgO}$ interfaces are not identical $[48,49]$. However, much more detailed studies are needed to elucidate the difference.

It may be noted that the hyperfine field of $30.8 \mathrm{~T}$ does not match with that of any of the known oxides of Fe. This rules out the possible oxidation of $\mathrm{Fe}$ in the interfacial region. However, hybridization of $\mathrm{O} 2 p$ and $\mathrm{Fe} 3 d$ levels has been predicted from first principle calculations [19]. The reduced hyperfine field component observed in the present case may represent those $\mathrm{Fe}$ atoms that are hybridized with oxygen. Any charge transfer between the orbitals would cause the magnetic moment and hence the hyperfine field to change. Thus, presence of an interfacial layer with a reduced hyperfine field of about $30 \mathrm{~T}$ provides evidence in favor of hybridization, as predicted by first principle calculations.

\section{CONCLUSIONS}

Growth of thin Fe film on $\mathrm{MgO}$ (001) substrate has been studied using in situ GISAXS and GINRS measurements. While GISAXS measurements provide information about morphological changes in the film, GINRS measurements provide information about the evolution of structural and magnetic properties with film thickness. The Fe film exhibits a Volmer-Weber type of growth. Formation of percolation islands takes place around a thickness of $2.0 \mathrm{~nm}$. A continuous film with bulklike properties is formed at a thickness of $4.0 \mathrm{~nm}$. Initially the islands are nonmagnetic in nature due to superparamagnetic relaxation. However, macroscopic percolation islands exhibit magnetic moment. The discontinuous film also exhibits a significant distortion from cubic symmetry, which may be attributed to possible strain in the film due to interfacial hybridization with oxygen and coalescence of islands. The film exhibits a weak PMA, which disappears once the structural distortion in the film disappears. Thus, PMA has its origin at least partly in structural distortions. Interfacial iron atoms are found to possess a lower hyperfine field as compared to bulk iron. Precise information about the interfacial layer is obtained using NRR measurements. It is found that about two monolayers of $\mathrm{Fe}$ at the interface have reduced hyperfine field, providing evidence in favor of hybridization with oxygen.

\section{ACKNOWLEDGMENTS}

Partial support for this paper was provided through Science and Engineering Research Board Project No. SB/S2/CMP$007 / 2013$. The travel grant for the experiment at Petra III, Germany, was provided by the Department of Science and Technology, Govt. of India. Fitting of GINRS spectra was done using the program REFTIM, kindly provided by Prof. Marina A. Andreeva.
[1] S. Ikeda, J. Hayakawa, Y. Ashizawa, Y. M. Lee, K. Miura, H. Hasegawa, M. Tsunoda, F. Matsukura, and H. Ohno, Appl. Phys. Lett. 93, 082508 (2008).

[2] J. Mathon and A. Umerski, Phys. Rev. B 63, 220403(R) (2001).

[3] X.-G. Zhang, W. H. Butler, and A. Bandyopadhyay, Phys. Rev. B 68, 092402 (2003).

[4] G. X. Miao, Y. J. Park, J. S. Moodera, M. Seibt, G. Eilers, and M. Münzenberg, Phys. Rev. Lett. 100, 246803 (2008).

[5] Y. Ke, K. Xia, and H. Guo, Phys. Rev. Lett. 105, 236801 (2010).

[6] C. Wang, A. Kohn, S. G. Wang, L. Y. Chang, S.-Y. Choi, A. I. Kirkland, A. K. Petford-Long, and R. C. C. Ward, Phys. Rev. B 82, 024428 (2010).

[7] S.-H. Yang, B. Balke, C. Papp, S. Döring, U. Berges, L. Plucinski, C. Westphal, C. M. Schneider, S. S. P. Parkin, and C. S. Fadley, Phys. Rev. B 84, 184410 (2011).

[8] J. W. Koo, S. Mitani, T. T. Sasaki, H. Sukegawa, Z. C. Wen, T. Ohkubo, T. Niizeki, K. Inomata, and K. Hono, Appl. Phys. Lett. 103, 192401 (2013).
[9] S.-M. Ahn and G. S. D. Beach, J. Appl. Phys. 113, 17C112 (2013).

[10] A. Kozioł-Rachwał, T. Giela, B. Matlak, K. Matlak, M. Ślęzak, T. Ślęzak, M. Zając, R. Rüffer, and J. Korecki, J. Appl. Phys. 113, 214309 (2013).

[11] J. Okabayashi, J. W. Koo, H. Sukegawa, S. Mitani, Y. Takagi, and T. Yokoyama, Appl. Phys. Lett. 105, 122408 (2014).

[12] S. Ikeda, K. Miura, H. Yamamoto, K. Mizunuma, H. D. Gan, M. Endo, S. Kanai, J. Hayakawa, F. Matsukura, and H. Ohno, Nat. Mater. 9, 721 (2010).

[13] S. Yakata, H. Kubota, Y. Suzuki, K. Yakushiji, A. Fukushima, S. Yuasa, and K. Ando, J. Appl. Phys. 105, 07D131 (2009).

[14] K. Yakushiji, T. Saruya, H. Kubota, A. Fukushima, T. Nagahama, S. Yuasa, and K. Ando, Appl. Phys. Lett. 97, 232508 (2010).

[15] D. C. Worledge, G. Hu, David W. Abraham, J. Z. Sun, P. L. Trouilloud, J. Nowak, S. Brown, M. C. Gaidis, E. J. O’Sullivan, and R. P. Robertazzi, Appl. Phys. Lett. 98, 022501 (2011). 
[16] Z. Wen, H. Sukegawa, S. Mitani, and K. Inomata, Appl. Phys. Lett. 98, 242507 (2011).

[17] M. Endo, S. Kanai, S. Ikeda, F. Matsukura, and H. Ohno, Appl. Phys. Lett. 96, 212503 (2010).

[18] C.-H. Lambert, A. Rajanikanth, T. Hauet, S. Mangin, E. E. Fullerton, and S. Andrieu, Appl. Phys. Lett. 102, 122410 (2013).

[19] H. X. Yang, M. Chshiev, B. Dieny, J. H. Lee, A. Manchon, and K. H. Shin, Phys. Rev. B 84, 054401 (2011).

[20] A. Manchon, C. Ducruet, L. Lombard, S. Auffret, B. Rodmacq, B. Dieny, S. Pizzini, J. Vogel, V. Uhlî́, M. Hochstrasser, and G. Panaccione, J. Appl. Phys. 104, 043914 (2008).

[21] A. Manchon, S. Pizzini, J. Vogel, V. Uhlîr, L. Lombard, C. Ducruet, S. Auffret, B. Rodmacq, B. Dieny, M. Hochstrasser, and G. Panaccione, J. Magn. Magn. Mater. 320, 1889 (2008).

[22] A. Hallal, H. X. Yang, B. Dieny, and M. Chshiev, Phys. Rev. B 88, 184423 (2013).

[23] K. H. He and J. S. Chen, J. Appl. Phys. 111, 07 C109 (2012).

[24] T. Nozaki, Y. Shiota, M. Shiraishi, T. Shinjo, and Y. Suzuki, Appl. Phys. Lett. 96, 022506 (2010).

[25] J. Balogh, I. Dézsi, Cs. Fetzer, J. Korecki, A. Kozioł-Rachwał, E. Młyńczak, and A. Nakanishi, Phys. Rev. B 87, 174415 (2013).

[26] S.-C. Lee, K.-S. Kim, S.-H. Lee, U.-H. Pi, K. Kim, Y. Jang, and U.-I. Chung, J. Appl. Phys. 113, 023914 (2013).

[27] K. Schlage, S. Couet, S. V. Roth, U. Vainio, R. Rüffer, M. M. Abul Kashem, P. Müller-Buschbaum, and R. Röhlsberger, New J. Phys. 14, 043007 (2012).

[28] G. Sharma, U. P. Deshpande, D. Kumar, and A. Gupta, J. Appl. Phys. 112, 023910 (2012).

[29] G. Sharma, A. Gupta, P. Rajput, and R. Rüffer, J. Phys. D: Appl. Phys. 47, 345305 (2014).

[30] J. Du, S. Gnanarajan, and A. Bendavid, Supercond. Sci. Technol. 18, 1035 (2005).

[31] T. Marukame, K. Matsuda, T. Uemura, and M. Yamamoto, Jpn. J. Appl. Phys. 44, 6012 (2005).

[32] H. Raanaei, H. Lidbaum, A. Liebig, K. Leifer, and B. Hjörvarsson, J. Phys.: Condens. Matter 20, 055212 (2008).

[33] H.-C. Wille, H. Franz, R. Röhlsberger, W. A. Caliebe, and F.-U. Dill, J. Phys.: Conf. Ser. 217, 012008 (2010).
[34] L. Z. Mezey and J. Giber, Jpn. J. Appl. Phys. 21, 1569 (1982); S. H. Overbury, P. A. Bertrand, and G. A. Somorjai, Chem. Rev. 75, 547 (1975).

[35] D. Babonneau, J. Appl. Cryst. 43, 929 (2010).

[36] R. Reitinger, B. Pfau, L.-M. Stadler, S. Stankov, M. Zajac, N. Spiridis, B. Sepiol, F. Zontone, and J. Korecki, Defect and Diffusion Forum 263, 177 (2007).

[37] J. F. Lawler, R. Schad, S. Jordan, and H. van Kempen, J. Magn. Magn. Mater. 165, 224 (1997).

[38] R. Röhlsberger, J. Bansmann, V. Senz, K. L. Jonas, A. Bettac, and K. H. Meiwes-Broer, O. Leupold, Phys. Rev. B 67, 245412 (2003).

[39] M. A. Andreeva, Hyperfine Interact. 185, 17 (2008).

[40] G. M. Bancroft and R. H. Platt in Advances in Inorganic chemistry and Radiochemistry, Vol. 15, edited by H. J. Emeleus and A. G. Sharpe (Academic Press, New York, 1972), p. 59.

[41] S. Mørup, J. A. Dumesic, and H. Topsøe in Applications of Mössbauer Spectroscopy, Vol. II, edited by R. L. Cohen (Academic Press, New York, 1980), p. 1.

[42] V. B. Naik, H. Meng, J. X. Xiao, R. S. Liu, A. Kumar, K. Y. Zeng, P. Luo, and S. Yap, Appl. Phys. Lett. 105, 052403 (2014).

[43] F. Spaepen, Acta Mater. 48, 31 (2000).

[44] A. Gupta, M. Gupta, S. Chakravarty, R. Rüffer, H.-C. Wille, and O. Leupold, Phys. Rev. B 72, 014207 (2005).

[45] S. Stankov, T. Ślęzak, M. Zając, M. Ślęzak, M. Sladecek, R. Röhlsberger, B. Sepiol, G. Vogl, N. Spiridis, J. Łażewski, K. Parliński, and J. Korecki in Mössbauer Spectroscopy: Applications in Chemistry, Biology, and Nanotechnology, edited by Virender K. Sharma, Göstar Klingelhöfer and Tetsuaki Nishida (John Wiley and Sons, New Jersey, 2013), p. 3.

[46] M. Zając, K. Freindl, K. Matlak, M. Ślęzak, T. Ślęzak, N. Spiridis, and J. Korecki, Surf. Sci. 601, 4305 (2007).

[47] S. Hine, T. Shinjo, and T. Takada, J. Phys. Soc. Jpn. 47, 767 (1979).

[48] S. G. Wang, R. C. C. Ward, T. Hesjedal, X.-G. Zhang, C. Wang, A. Kohn, Q. L. Ma, Jia Zhang, H. F. Liu, and X. F. Han, J. Nanosci. Nanotechnol. 12, 1006 (2012).

[49] E. Młyńczak, K. Freindl, N. Spiridis, and J. Korecki, J. Appl. Phys. 113, 024320 (2013). 